Die Zusammensetzung des Anteiles 1 entspricht einem normalen PortlandZementklinker, die des Anteiles 2 einer Hochofenschlacke.

Da die Berechnung der Hochofenschlacke sich auf den Gehalt an SulfidSchwefel in den drei untersuchten Proben gründet, und da jener relativ gering ist, so muß die Bestimmung des Sulfid-Schwefels mit äuberster Präzision ausgeführt werden, denn schon ein kleiner Fehler hierbei verschiebt das Endresultat erbeblich.

Die Schwebeanalyse liefert in der Hand eines erfahrenen und gewissenhaften Chemikers befriedigende Resultate. Sie versagt natürlich, wenn dem Portland-Zement zwei oder mehrere Schlackenarten beigemischt sind, die einen verschiedenen Gehalt an Sulfid-Schwefel aufweisen.

Doch wird man meistens, schon bei der mikroskopischen Prüfung der abgeschiedenen Schlacke, also der Trennung 4 erkennen, ob eine einheitliche Schlacke oder mehrere Schlackenarten vorliegen,

\title{
Hat sich bei der Durchfülurung des Nahrungsmittelgesetzes rom 14. Mai 1879 das Bedürfnis herausgestellt, wesentliche Bestimmungen dieses Gesetzes zo ändern?
}

Von

\section{A. Juckenack in Berlin.}

„Die Notwendigkeit, die Nahrungsmittelgesetzgebung auszugestalten und insbesondere bindende Vorschriften über die Zusammensetzung und die Beurteilung der Nahrungsmittel und weiter darüber zu geben, nach welchen Methoden bei der amtlichen Untersuchung von Nahrungsmitteln vorzugehen ist" ${ }^{ \pm}$), hat die Regierung wiederholt im Reichstage anerkannt. Dem Vorwort $\mathrm{zu}$ den bisher vom Kaiserlichen Gesundheitsamte herausgegebenen Entwürfen zu Festsetzungen über Lebensmittel ist zu entnehmen, daß die Absicht besteht, durch Änderung des Nahrungsmittelgesetzes vom 14. Mai 1879 gesetzliche Unterlagen zum Erlab von Verordnungen zu schaffen, die für das ganze Reich rechtsverbindlich sein und das Feilhalten und Verkaufen verfälschter, nachgemachter, verdorbener und irreführend bezeichneter minderwertiger Lebensmittel behandeln sollen. Zu diesem Bestreben der Reichsregierung habe ich bereits in einem auf der XII. Hauptversammlung des Vereins Deutscher Nahrungsmittelchemiker am 6. Juni 1913 in Breslau gehaltenen Vortrag ${ }^{2}$ ) Stellung genommen, auf den ich hiermit insoweit Bezug nehme. Ob die Reichsregierung zur Erreichung ihres Zieles demnächst eine Umgestaltung und Erweiterung der $\S \S 5,8,10$ und 11 des bisherigen Gesetzes oder einen anderen Weg wählen wird, ist noch nicht bekannt, hat zudem zurzeit kein besonderes Interesse.

Der Erklärung des Bundesratskommissars vom 18. März 1911 ist aber außerdem zu entnehmen, daß die Reichsregierung die bisherige Nahrungsmittelgesetzgebung noch weiter auszugestalten gedenkt, wenn auch den wirtschaftlich wichtigsten Teil der von ihr beabsichtigten Änderungen die rechtsverbindlichen Verordnungen der ange-

1) Aus der Erklärung des Bundesratskommissars in der Reichstagssitzung vom 18. März 1911.

3) Diese Zeitschrift 1913, 26, 488. 
gebenen Art bilden dürften. Darüber aber, welche Anderungen des Nahrungsmittelgesetzes auferdem noch in Aussicht genommen worden sind, ist bisher nichts bekannt geworden.

In der Presse der beteiligten Verkehrskreise sind in Laufe der Jahre wiederholt Wünsche und Forderungen geltend gemacht worden, die Änderungen des Gesetzes vom 14. Mai 1879 anstreben. Sie hier zusammenzutragen und zu besprechen, würde zu weit führen. Der Zweok dieser Zeilen ist lediglich der, aus den in der Praxis gesammelten Erfahrungen heraus die Frage zu erörtern, ob und inwieweit etwa sich bei der Durchführung des Nahrungsmittelgesetzes das Bedürfnis herausgestellt hat, wesentliche Bestimmungen dieses Gesetzes $\mathrm{zu}$ ändern. Um diese Erörterungen übersichtlicb gestalten zu können, werde ich $\mathrm{zu}$ den einzelnen Paragraphen Stellung: nehmen. Ich gestatte mir, hierbei zur Erläuterung auf einen von mir bearbeiteten kleinen Kommentar zum Nahrungsmittelgesetz zu verweisen, der unter der Uberschrift "Die reichsgesetzliche Regelung des Verkehrs mit Nahrungsmitteln, GenuBmitteln und Gebrauchsgegenständen"zurzeit im 2. Teil des III. Bandes der "Chemie der menschlichen Nahrungs- und Genuhmittel" von Geheimrat Prof. Dr. phil. et Dr. ing. h. c. J. König erscheint.

\section{$\mathrm{Zu} \S 1$.}

Bisher unterliegt nur der Verkehr mit Nahrungs- und GenuBmitteln, sowie mit Spielwaren, Tapeten, Farben, EB-, Trink- und Kochgeschirr und mit Petroleum der polizeilichen Beaufsichtigung. Die Bekleidungsgegen stände hat der Gesetzgeber absichtich nicht der polizeilichen Kontrolle unterworfen, obwohl er sie in $\$ 5$ Ziff. 4 und $\$ 12$ Abs. 1 Ziff. 2 berücksichtigt hat. Geht man die später erlassenen Sondergesetze durch, so begegnet man verschiedenen Gegenständen, an die demnächst zum Schutze der Gesundheit bestimmte Anforderungen gestellt worden sind, die aber bisher noch nicht ausdrücklich der polizeilichen Beaufsichtigung nach $\S \S 2,3$ des Nahrungsmittelgesetzes unterworfen worden sind. Es kann natürlich nicht zweifelhaft sein, daß die Polizei schon zurzeit das Recht hat, Proben dieser sowie der hier sonst noch interessierenden Gegenstände zu ,k a u fen" und, falls der Verkauf verweigert werden sollte, nach Maßgabe der Bestimmungen der Strafprozeßordnung (vergl. insbesondere $\S \S 94,98$ ) zu beschlagnahmen, auch bieten ibr unter Umständen landesrechtliche Bestimmungen nach dieser Richtung weitgehende Befugnisse, doch dürfte sie zurzeit nicht in der Lage sein, Proben derartiger Gegenstände auf Grund des Reichsgesetzes vom 14. Mai 1879 zu ,entnebmen". Nach dieser Richtung kommen u. a. in Betracht: Flüssigkeitsmabe, Druckvorrichtuagen zum Ausschank von Bier, Siphons, Saugringe, Warzenhütchen, Leitungen für Bier, Wein und Essig, Gesehirre und Gefäße zur Verfertigung von Getränken und Fruchtsäften, Gefäße zur Aufbewahrung von Getränken, Metallfolien zur Packung von Schnupf-und Kautabak sowie von Käse, Müblsteine zur Verfertigung von Nahrungs- und Genubmitteln, Gefäbe, Umhüllungen und Schutzbedeckungen zur Aufbewahrung und Verpackung von Nahrungs- und Genußmitteln, kosmetische Mittel (einschl. Mittel zur Pflege usw. der Nägel - Gesetz vom 14. Juni 1912-), Blumentopfgitter, künstliche Christbäume, Möbelstoffe, Teppiche, Stoffezu Vorhängen und Bekleidungsgegenständen, Masken, Kerzen, künstliche Blätter, Blumen und Früchte, Schreibmaterialien, Lampen- 
und Lichtschirme sowie Lichtmanschetten und Oblaten (vergl. die Reichsgesetze, betreffend den Verkehr mit blei- und zinkhaltigen Gegenständen, vom 25. Juni 1887 und betreffend die Verwendung gesundheitsschädlicher Farben bei der Herstellung von Nahrungsmitteln, Genußmitteln und Gebrauchsgegenständen, vom 5. Juli 1887). Es kann vorläufig dahin gestellt bleiben, ob es erforderlich ist, den Verkehr mit allen diesen Gegenständen schlechthin der polizeilichen Beaufsichtigung zu unterwerfen. Immerhin bedarf aber offensichtlich $\$ 1$ N.M.G. einer weiteren Fassung. Zweckmäßig dürfte es sein, diese möglichst allgemein zu wählen, zumal sich die Beaufsichtigung in der Praxis zweifellos fast ausschließlich auf solche Gegenstände erstrecken wird, an die gesetzlich bestimmte Anforderungen gestellt werden. Denn bekanntlich kann nach $\S 2$ des Strafgesetzbuches eine Handlung nur dann mit Strafe belegt werden, wenn diese Strafe gesetzlich bestimmt war, bevor diese Handlung begangen wurde (nulla poena sine lege!). Hinzu kommt, daß sich die Nahrungsmittelgesetzgebung noch immer im Ausbau befindet. Z. B. werden künftig nicht nur die Leitungen sondern auch die Trichter für Essig, und nicht nur die Metallfolien zur Packung von Schnupftabak, Kautabak und Käse, sondern auch die zur Packung von Schokolade und anderen Lebensmitteln in der Gesetzgebung zu berücksichtigen sein. Zudem wird allgemein an die zur Verfertigung und Aufbewahrung von Nahrungs- und Genußmitteln bestimmten Geschirre und Gefäße zu denken sein. Ferner fehlen bisher in $\S 1$ gänzlich lebende Tiere, die zu dem $Z$ weck feilgehalten und verkauft werden, alsbald getötet zu werden und als menschliches Lebensmittel Verwendung zu finden. Diese Materie ist durch die Schlachtvieh- und Fleischbeschau-Gesetzgebung nicht erschöpfend geregelt worden; ich erinnere nur an den Verkehr mit lebenden Fischen, lebendem Federvieh, Krebsen, Muscheln, Schnecken usw. Endlich ist auch die Frage noch nicht hinreichend geklärt, ob die Riechmittel zu den GenuBmitteln im Sinne des Nahrungsmittelgesetzes gehören. Infolgedessen erwähnt sie das Branntweinkontingentsgesetz vom 14. Juni 1912 in $\S 21$ neben den Genußmitteln, kosmetischen Mitteln usw. besonders.

\section{$\mathrm{Zu} \& 2$.}

Die Befugnis der Polizei, nur die für den Verkehr mit dem Publikum bestimmten Räumlichkeiten zu betreten, reicht erfahrungsgemäß nicht aus; zudem ist es erforderlich, hier auch den zuständigen Sachverständigen der Polizei entsprechende Befugnisse einzuräumen und zugleich ihre insoweit in Betracht kommende Vereidigung im Gesetz anzuordnen. Diese Mängel sind bereits in den Sondergesetzen über den Verkehr mit Wein sowie mit Butter, Käse, Schmalz und deren Ersatzmitteln im Rahmen dieser Gesetze berücksichtigt worden. Allerdings kann die Polizei ihren Sachverständigen entsprechende Beamteneigenschaft beilegen, doch ist dies im Interesse der Tätigkeit der Sachverständigen nicht zweckmäßig. Andererseits können Industrie und Handel es nur begrüßen, wenn die Kontrollen, soweit es sich nicht um einfache Fragen handelt, unter Zuziehung von Sachverständigen vorgenommen werden. Die Beamten und Sachverständigen der Polizei müssen zudem befugt sein, in den in Betracht kommenden Räumlichkeiten einfache, cinen erheblichen Zeitaufwand nicht erfordernde Prüfungen der in Betracht kommenden Gegenstände vorzunehmen.

Um die bei der Durchführung des Nabrungsmittelgesetzes hervorgetretenen Mängel zu beseitigen, sehen neuere preußische Polizeiverordnungen, betreffend den 
gewerblichen und Handelsverkehr mit Nahrungs- und Genußmitteln ${ }^{1}$, folgende Bestimmungen vor:

„Außer dem Verkehr mit Nahrungs- und Genußmitteln nach Mabgabe des Gesetzes vom 14. Mai 1879 (R.G.BL. S. 145 fg.) unterliegen auch die Zubereitung, die Aufbewabrung, das Ausmessen, das Auswägen und die Beförderung der Nahrungs- und GenuBmittel der polizeilichen Beaufsichtigung und demgemäB auch alle Räumlichkeiten, Einrichtungen und Geräte, welche der Zubereitung, der Aufbewahrung, dem Ausmessen, dem Auswägen und der Beförderung derselben dienen.

Die Beamten und Sachverständigen der Polizei sind daher befugt, alle nach Absatz 1 in Betracht kommenden Räumlichkeiten während der ortsüblichen Geschäftszeit und, wenn der Betrieb zu einer anderen Zeit ausgeübt wird, z. B. in Bäckereien, auch innerhalb dieser Betriebszeit zu betreten. Die Inhaber dieser Räumlichkeiten sind verpflichtet, den Eintritt in sie, die Entnahme einer Probe oder die Revision zu gestatten."

\section{$\mathrm{Zu} \& 3$}

Durch Ausbau des $\S 2$ im Sinne vorstehender Ausführungen erübrigen sich die bisherigen Bestimmungen in $\S 3$. Es wird aber $z u$ prüfen sein, ob im öffentlichen Interesse nicht Maßnahmen gegen solche Personen vorgesehen werden müssen, die das Gesetz in gemeingefährlicher oder ekelerregender Weise verletzt oder sich wiederholt vorsätzlich gegen das Gesetz vergangen haben.

Bekanntlich ist schon wiederholt und von verschiedenen Seiten angeregt worden, durch Änderung der Reichsgewerbeordnung weitere Lebensmittelbetriebe, z. B. den Milchhandel und Wurstfabriken, konzessionspllichtig zu machen, damit die Eignung der Räumlichkeiten und die Zuverlässigkeit der Betriebsinhaber geprüft werden kann (vergl. z. B. R. Abel, Handbuch der praktischen Hygiene, S. 453/454). Es liegen aber bisher noch keine Anhaltspunkte dafür vor, ob dieser Weg einmal beschritten werden wird. $\mathrm{Zu}$ erörtern dürfte daher die Frage sein, ob in $\$ 3$ nicht Bestimmungen vorzusehen sind, die den Gerichten das Recht verleihen, vorgenannten Personen unter Strafandrohung zu verbieten, auf die Dauer von etwa einem bis fünf Jahren ein Lebensmittelgeschäft zu betreiben, zu leiten oder in einem Lebensmittelgeschäft tätig zu sein.

\section{$\mathrm{Zu} \S 4$.}

Ein Bedürfnis, den Inhalt dieser Bestimmungen zu ändern, hat sich bisher nicht ergeben. Es sei aber hervorgehoben, daß diese Bestimmungen unbedingt aufrecht erhalten bleiben müssen, weil die gesamte einschlägige Materie lediglich reichsgesetzlich nicht geregelt ist und zudem nicht geregelt werden kann. (Vergl, auch die Ausführungen zu $\$ 2$ ).

\section{$\mathrm{Zu} \$ 5$}

Mit Rücksicht auf die in Aussicht genommenen für das ganze Reich rechtsverbindlichen Verordnungen wird $\S 5$ vollständig umzugestalten sein. Denn diese Verordnungen sind bekanntlich nicht nur zum Schutze der Gesundheit, sondern auch zum Schutze gegen Täuschungen und wirtschaftliche Benachteiligungen gedacht. Hier auf sie näber einzugehen, erübrigt sich mit Rücksicht auf meine früheren einschlägigen Ausführungen. Es bleibt zudem zunächst noch abzuwarten, ob in dieser Weise auch

$\left.{ }^{1}\right)$ Vergl. Gesetze und Verordnungen (Beilage zu dieser Zeitschrift) 1912, 5, 132-135. 
eine Regelung des Verkehrs mit Gebrauchsgegenständen beabsichtigt ist. Bei dieser umfassenden Änderung wird der Verkehr mit Lebensmitteln in Konsumvereinen im Auge zu behalten sein. Denn nach der Rechtsprechung findet in Konsumvereinen, die ledighich eine distributive Tätigkeit ausüben, kein ,gewerbs mäßiges“ Feilhalten und Verkaufen statt. Infolgedessen genießen diese Vereine dem Kleinhandel gegenüber Vorteile (vergl. z. B. $\S 4$ des Margarinegesetzes vom 15. Juni 1897 und $\S \S 6,19$ des Weingesetzes vom 7. April 1909), deren Berechtigung mindestens einer Erörterung bedürftig zu sein scheint.

Daß schließlich im $\S 5$ noch die Ermächtigung des Bundesrates zur Aufstellung von Grundsätzen, nach denen die erforderlichen technischen Untersuchungen auszuführen sind, vorgesehen werden mübte, sei nur nebenbei bemerkt.

\section{$\mathrm{Zu} \S 6$.}

$\S 6$ gibt bisher die Möglichkeit, im Verordnungswege das gewerbsmäßige Herstellen, Verkaufen und Feilhalten von Gegenständen, die zur Fälschung von Nahrungsoder Genußmitteln bestimmt sind, zu verbieten oder zu beschränken. Es wird demnächst zu prüfen sein, ob dieses Verordnungsrecht nicht auch auf das Ankündigen, Ansichbringen und Verkaufsvermitteln solcher Stoffe auszudehnen sein wird, deren Verwendung bei der Herstellung, Behandlung oder Bearbeitung bestimmter Nahrungsoder Genußmittel schlechthin unzulässig ist (vergl. auch $\S 26$ Abs. 1 Ziff. 3 des Weingesetzes vom 7. April 1909).

\section{$\mathrm{Zu} \S 7$.}

$\S 7$ ordnet an, daß die auf Grund der $\S \S 5$ und 6 erlassenen Kaiserlichen Verordnungen dem Reichstage vorzulegen sind und gibt dem Reichstage zugleich das Recht, zu verlangen, daß die ihm nicht genehmen Verordnungen außer Kraft gesetzt werden. Ähnliche Bestimmungen enthalten die Sondergesetze über den Verkehr mit Butter, Wein, Fleisch usw. hinsichtlich des Verordnungsrechts, das sie dem Bundesrat eingeräunt haben, nicht. Sollte es in größerem Umfange zu einer Regelung des Verkehrs mit Lebensmitteln durch Erlaß einschlägiger Verordnungen kommen, so wird zu prüfen sein, ob es zweckmäßig sein wird, $\S 7$ in seiner bisherigen Fassung aufrecht zu halten. Der Reichstag ist bisher nicht verpflichtet, über die Verordnungen zu verhandeln. Die Verordnungen vor ihrem Erlab dem Reichstage vorzulegen, würde ihren $Z$ weck, in beweglicher Form der Veränderlichkeit der Verhältnisse im Verkebr Rechnung zu tragen, vereiteln und der umständlichen Schaffung zahlreicher und nur schwer zu ändernder Sondergesetze gleichkommen. Werden andererseits einschneidende Verordnungen erlassen und ist demnächst, wenn der Reichstag zusammentritt, mit einer Außerkraftsetzung und dann wieder mit dem Erlaß neuer Vorschriften zu rechnen, so wird eine dauernde Rechtsunsicherheit geschaffen, die ohne Zweifel erhebliche wirtschaftliche Schäden zur Folge haben wird. Denn erfahrungsgemäß werden Lebensmittel-Industrie und -Handel durch gewisse Härten in Gesetzen und Verordnungen bei weitem nicht so geschädigt als durch unklare Vorschriften und Rechtsunsicherheit. Hinzukommt, daB der Bundesrat selbstverständlich niemals Verordnungen in Kraft setzen wird, die nicht sehr sorgfältig beraten worden sind und dem Geiste der deutschen Nahrungsmittelgesetzgebung nicht entsprecben. Mithin dürfte es am zweckmäßigsten sein, dem Bundesrat auf dem hier in Betracht kommenden Gebiete reichsrechtlich ähnliche Befugnisse einzuräumen, wie er sie zurzeit schon auf Grund mehrerer Gesetze besitzt und wie sie landesrechtlich schon seit langer Zeit 
den Polizeiverwaltungen eingeräumt worden sind. Hierdurch würde zugleich eine nicht unbedenkliche Buntscheckigkeit der Lebensmittelpolizeiverordnungen vermieden.

\section{$\mathrm{Zu} \S 8$.}

Sollten die in Aussicht genommenen Verordnungen den Charakter von Ausführungsbestimmungen zu $\$ \S 10-14$ erhalten, so werden die bisher in $\$ 8$ vorgesehenen Strafen nicht ausreichen und mit den in $\$ \S 10,12,13,14$ nicht im Einklang stehen. Es bleibt daher zunächst abzuwarten, welcber Weg grundsätzlich eingeschlagen werden wird (vergl. die allgemeinen einleitenden Ausfübrungen).

\section{$\mathrm{Zu} \$ 9$.}

Hier dürfte zu prüfen sein, ob nicht eine absichtliche Veränderung von amtlich verschlossenen oder versiegelten Gegenproben, die dem Besitzer gemäß § 2 auf Verlangen zurückzulassen sind, mit angemessener Strafe bedroht werden muß. Im Interesse der Rechtspflege dürfte es aber vielleicht noch zweckmäßiger sein, anzuordnen, daß die sogenannten Gegenproben amtlich zu asservieren und auf Antrag des Besitzers amtlich den von ihm benannten Sachverständigen zu äbersenden sind.

$$
\mathrm{Zu} \$ \$ 10-14 \text {. }
$$

Hinsichtlich dieser Bestimmungen möchte ich auf das Vorwort zu den von Kaiserlichen Gesundheitsamte herausgegebenen Entwürfen zu Festselzungen über Lebensmittel und die von mir auf der diesjährigen Versammlung des Vereins Deutscher Nahrungsmittelchemiker in Breslau gemachten Ausführungen verweisen, um nicht bereits Bekanntes wiederholen zu müssen. Jedenfalls dürfen hier die Regelung des Verkehrs mit irreführend bezeichneten minderwertigen Lebensmitteln und die Bestimmungen in $\$ 367$ Ziff. 7 des Strafgesetzbuches nicht überseben werden. Zu beachten ist auch noch, daß $\$ 12 \mathrm{Abs} .1$ Ziff. 2 daraufhin $z u$ prüfen sein wird, inwieweit er etwa mit Rücksicht auf die zu $\$ 1$ gemachten Ausführungen zu ergänzen bzw. zu erweitern sein wird.

\section{$\mathrm{Zu} \S 15$.}

Mit Rücksicht auf die bisherige Rechtsprechung des Reichsgerichts wird hier eine dem $\S 31$ Abs. 3 des Weingesetzes rom 7. April 1909 sowie dem $\$ 28$ Abs. 2 des Branntweinkontingentgesetzes vom 14. Juni 1912 entsprechende Vorschrift aufzunehmen sein, die die Einziehung auch dann anordnet oder ermöglicht, wenn die Strafe gemäß \& 73 des Strafgesetzbuches nicht auf Grund des Nahrungsmittelgesetzes zu bestimmen ist. Ferner wird zum Ausdruck zu bringen sein, dal die Bestimmungen der $\$ \$ 40,42$ des Strafgesetzbuches (z. B. dal Gegenstände, die zur Begehung eines vorsätzlichen Vergehens oder Verbrechens gebraucht oder bestimmt sind, eingezogen werden können, sofern sie dem Täter oder Mittäter gehören) durch das Nabrungsnittelgesetz unberührt bleiben, weil das Reichsgericht bisher die Ansicht vertreten hat, $\$ 15$ N.M.G. habe die hier in Betracht lrommende Materie erschöpfend geregelt, daher sei $\S \S 40,42$ St. G. B. neben $\S 15$ N.M. G. nicht anwendbar. Diese Auffassung hat schon wiederholt zu Bedenken Anlaß gegeben (z. B. bei Anträgen auf Einziehung von Maschinen, die zur Herstellung verfälschter Butter gebraucht worden waren.)

\section{Zu $\& 16$.}

Da nach Ansicht des Reichsgerichtes die Veröffentlichung des Strafurteils eine Nebenstrafe und daher nicht $\mathrm{zu}$ erkennen ist, wenn im Falle ideellen Zusammen. treffens die Strafe nicht aus dem Nahrungsmittelgesetz zu erkennen ist, während 
nach den Motiven zu letztgenanntem Gesetz die Veröffentlichung nicht den Charakter einer Nebenstrafe im eigentlichen Sinne haben, sondern nur eine besonders verstärkte Bekanntmachung des ohnehin für die öffentliche Verkündung bestimmten Strafurteils darstellen sollte, schreibt $\$ 28 \mathrm{Abs} .2$ des Branntweinkontingentsgesetzes vom 14. Juni 1912 vor, daß die Veröffentlichung auch dann zulässig sein soll, wenn die Strafe gemäB $§ 73$ des Strafgesetzbuches auf Grund eines andern Gesetzes zu bestimmen ist. Eine entsprechende Vorsehrift dürfte auch in $\$ 16$ Abs. 1 N.M.G. aufzunehmen sein, um den Willen des Gesetzgebers klar zum Ausdruck zu bringen.

In $\S 16$ Abs. 4 ist nicht erwähnt worden, daß dem rechtskräftig Verurteilten auch die Kosten zur Last fallen, die durch die notwendige polizeiliche Untersuchung weiterer Überführungsstücke (z. B. von Milch-Stallproben, Proben aus Packungen des Lieferanten usw.) erwachsen sind. Allerdings kommen zurzeit insoweit noch $\$ \S 161$, 497 der Strafprozebordnung in Betracht, doch dürfte es zweckmäbig sein, diese Kostenfrage in bezug auf das Nahrungsmittelgesetz und seine Ergänzungsgesetze in $\S 16$ Abs. 4 tunlichst erschöpfend zu regeln. Hierzu dürfte auch schon deswegen Anlaß vorliegen, weil der Abs. 4 dem $\$ 16$ erst durch das Sondergesetz vom 29. Juni 1887 eingefügt worden ist und man damals (vergl. die Motive zu diesem Gesetz ${ }^{1}$ )) anscheinend an diese notwendigen durch das Verschulden des Verurteilten erwachsenen Kosten nicht gedacht bat, weil damals noch nicht hinreichend einschlägige Erfahrungen vorlagen. Aber auch die der Polizei durch Ankauf von Proben erwachsenen Kosten sollten hier besonders berücksichtigt werden, zumal nicht alle Proben im Sinne des $\S 2$ des Gesetzes entnommen werden.

\section{$\mathrm{Zu} \S 17$.}

Die Bundesstaaten haben bekanntlich zwecks Durchführung einer ausreichenden Kontrolle und zur Erleichterung der den Gemeinden oder Gemeindeverbänden hierdurch entstehenden Kosten auf die ihnen zustehenden Geldstrafen zugunsten der Kassen verzichtet, welche die Kosten zur Unterbaltung offentlicher Anstalten zur technischen Untersuchung ron Nahrungs- und Genußomitteln im Sinne dieses Gesetzes tragen. Denn die Überwachung des Verkehrs mit Nabrungsmitteln ist Aufgabe der örtlichen Polizeiverwaltungen. Also nicht den genannten Anstalten, sondern den genannten Kassen und somit Verbänden fließen die Geldstrafen, soweit sie von Verurteilten gezahlt werden, zu. Dieser Sachverhalt ist schon wiederholt verkannt worden und hat daher schon wiederholt Anlaß zu der Anregung gegeben, die Bestimmungen des $\$ 17$ aufzuheben. Es ist aber nicht zu übersehen, daß hierdurch die Gemeinden einen erheblichen Ausfall erleiden würden und daß infolgedessen eine entsprechende Einschränkung der Nahrungsmittelkontrolle zu besorgen ist, die offensichtlich nicht mehr dem Willen des Gesetzgebers entsprechen würde. Es wird auch kein Unbefangener den öffentlichen unabhängigen Anstalten zutrauen, daß sie etwa auf die Verurteilungen zu möglichst hohen Geldstrafen Wert legen oder gar derartige Verurteilungen anregen; Zudem hängt bekanntlich das Urteil lediglich von dem pflichtmäßigen Ermessen deutscher Gerichte ab. Mithin wird man derartigen Anregungen im Interesse der Allgemeinheit nicht stattgeben können.

1) Diese Zeitschrift 1908, 16, 137-139. 